\title{
Prevalência, severidade e fatores associados à depressão em estudantes universitários*
}

Larissa Barreto dos Santos ${ }^{1,2}$

(D) https://orcid.org/0000-0001-5587-4264

Karina Gomes do Nascimento ${ }^{1,3}$

(D) https://orcid.org/0000-0002-6606-0474

Andréia Guedes Oliva Fernandes ${ }^{1}$

(D) https://orcid.org/0000-0001-5584-5658

Talita de Cássia Raminelli-da-Silva

(D) https://orcid.org/0000-0002-9181-8478

Apoio financeiro da FAP-DF, Unieuro

${ }^{1}$ Centro Universitário Euro-Americano, Águas Claras, Brasília, DF, Brasil.

${ }^{2}$ Bolsista da Fundação de Apoio a Pesquisa do Distrito Federal, Brasília, DF, Brasil.

${ }^{3}$ Bolsista do Centro Universitário Euro-Americano, Brasília, DF, Brasil
Objetivo: identificar a prevalência, a severidade e os fatores associados à depressão entre estudantes universitários no Distrito Federal. Método: estudo transversal realizado com 521 estudantes universitários, com idade entre 18 e 60 anos. Os sintomas depressivos foram avaliados através da escala Patient Health Questionnaire-9 (PHQ-9). Utilizou-se estatística descritiva e o teste do qui-quadrado. Resultados: dentre os 521 indivíduos estudados, houve predomínio do sexo feminino. A prevalência de sintomas depressivos foi de 521 (96,6\%) estudantes universitários, sendo 31,3\% com depressão suave, 23,4\%, depressão mínima, 13,1\% depressão moderadamente grave, 9,6\% depressão grave e 9,2\% depressão moderada. A renda familiar e o semestre cursado são fatores associados para a severidade da depressão. Conclusão: a prevalência da depressão no ambiente universitário é comum, sendo uma proporção alta e preocupante. É crucial o rastreamento da depressão, sua severidade e seus fatores associados, a fim de estimular reflexão sobre a temática e pensar em estratégias de intervenção para a prevenção e promoção da saúde mental.

Descritores: Depressão; Estudantes; Universidades; Saúde Mental.

\section{Como citar este artigo}

Santos LB, Nascimento KG, Fernandes AGO, Raminelli-da-Silva CR. Prevalence, severity and factors associated with depression in university students. SMAD, Rev Eletrônica Saúde Mental Álcool Drog. 2021 jan.- mar.;17(1):92-100. doi: https://dx.doi.org/10.11606/issn.1806-6976.smad.2021.167804 


\title{
Prevalence, severity and factors associated with depression in university students
}

\begin{abstract}
Objective: to identify the prevalence, severity and factors associated with depression among university students in the Federal District. Method: a cross-sectional study carried out with 521 university students, aged between 18 and 60 years old. Depressive symptoms were assessed using the Patient Health Questionnaire-9 (PHQ-9) scale. Descriptive statistics and the chi-square test were used. Results: among the 521 individuals studied, there was predominance of females. The prevalence of depressive symptoms was 521 (96.6\%) university students; 31.3\% with mild depression; 23.4\%, minimal depression, 13.1\%, moderately severe depression; 9.6\%, severe depression; and 9.2\%, moderate depression. Family income and the semester attended are associated factors for the severity of depression. Conclusion: the prevalence of depression in the university environment is common, being at a high and worrying proportion. It is crucial to track depression, its severity and its associated factors, in order to stimulate reflection on the theme and to think of intervention strategies for the prevention and promotion of mental health.
\end{abstract}

Descriptors: Depression; Students; Universities; Mental Health.

\section{Prevalencia, severidad y factores asociados a la depresión en estudiantes universitarios}

Objetivo: identificar la prevalencia, la gravedad y los factores asociados a la depresión entre los estudiantes universitarios del Distrito Federal. Método: estudio transversal realizado con 521 estudiantes universitarios, con edades comprendidas entre 18 y 60 años. Los síntomas depresivos se evaluaron mediante la escala del Patient Health Questionnaire-9 (PHQ-9). Se utilizó estadística descriptiva y la prueba de chi-cuadrado. Resultados: Entre los 521 individuos estudiados, predominó el sexo femenino. La prevalencia de síntomas depresivos fue de 521 (96.6\%) estudiantes universitarios, 31.3\% con depresión leve, 23.4\%, depresión mínima, 13.1\% depresión moderadamente severa, 9.6\% depresión severa y 9, 2\% de depresión moderada. Los ingresos familiares y el semestre asistido son factores asociados para la gravedad de la depresión. Conclusión: la prevalencia de depresión en el entorno universitario es común, siendo una proporción alta y preocupante. Es crucial rastrear la depresión, su gravedad y sus factores asociados, para estimular la reflexión sobre el tema y pensar en estrategias de intervención para la prevención y promoción de la salud mental.

Descriptores: Depresión; Estudiantes; Universidades; Salud Mental. 


\section{Introdução}

A depressão é uma doença psiquiátrica crônica, que afeta mais de 300 milhões de pessoas em nível mundial(1), crescente na população universitária(2-10). É caracterizada por tristeza grave ou persistente, que interfere na vida pessoal e, muitas vezes, diminui o interesse ou o prazer nas atividades diárias do indivíduo. Dentre as possíveis etiologias desta doença, pode-se citar, os fatores biológicos e os psicossociais ${ }^{(11)}$.

São exemplos destes fatores que influenciam no surgimento da depressão, comumente evidenciados no contexto universitário, a dificuldade em conciliar vida pessoal e acadêmica, o prejuízo no sono, o sentimento de culpa, a autoconfiança reduzida, a fadiga, a insatisfação com o curso, os conflitos familiares, os problemas com os relacionamentos sociais e interpessoais, a renda salarial baixa, o desemprego, o sedentarismo e a solidão(12-15).

Apesar de ser o mal do século, ainda há muito preconceito, tabu e desinformação relacionados à depressão, pois o prognóstico é fornecido através das informações clínicas do paciente e com a utilização de escalas, para auxiliar no diagnóstico, sem a necessidade de exames complementares. Isso faz com que, muitas pessoas desacreditem na existência da doença e, algumas vezes, a injúria é confundida com preguiça, desânimo, falta de interesse, o que causa mais sofrimento para o paciente ${ }^{(16)}$.

É reconhecido que a depressão dos estudantes, no decorrer da graduação, pode acarretar em consequências nas diversas esferas da vida, tais como, a pessoal, a familiar, a profissional e a acadêmica. No âmbito acadêmico, são características, observadas nos estudantes diagnosticados com depressão, a queda do rendimento, o aumento do risco de abandono/desistência do curso e o aumento do risco de suicídio(14,17).

Tendo em vista, a alta prevalência da depressão no mundo(1-10) e o seu impacto negativo, em múltiplos aspectos da vida, em especial, na da população universitária(18-20), acredita-se que, a identificação precoce da doença, sua gravidade e seus fatores associados, são cruciais para o reconhecimento do impacto dessa enfermidade pela população, pelos profissionais de saúde e pelos órgãos governamentais, responsáveis pela gestão de políticas públicas de saúde, de modo a estimular a reflexão acerca da temática e instigar o desenvolvimento de estratégias/ações voltadas para prevenção da doença e para a promoção de saúde mental, a exemplo, no âmbito universitário(13,21-24). Assim, objetiva-se com este estudo, identificar a prevalência, a severidade e os fatores associados à depressão, em estudantes de uma Instituição de Ensino Superior (IES) privada no Distrito Federal.

\section{Método}

\section{Desenho / local /período do estudo}

Trata-se de um estudo transversal de caráter descritivo, realizado em uma IES privada, localizada em Brasília, no Distrito Federal, Brasil. Nesta Instituição de Ensino estavam matriculados, no período da coleta de dados, do atual estudo, 5.507 estudantes das diferentes áreas do conhecimento, as quais incluem Ciências da Saúde; Ciências Exatas e Ciências Humanas. A coleta de dados ocorreu no período entre abril e maio de 2019.

\section{Seleção da amostra}

Foram investigados estudantes entre 18 e 60 anos, regularmente matriculados na IES e utilizou-se uma amostra de conveniência.

\section{Coleta de dados}

Utilizou-se um questionário estruturado elaborado pelas próprias pesquisadoras para a coleta dos dados sociodemográficos. Para avaliar a frequência de sinais e sintomas de depressão nas últimas duas semanas, utilizou-se o questionário Patient Health Questionnaire-9 (PHQ-9). O mesmo foi validado no Brasil para uso na população adulta e contém 9 questões, dispostas em uma escala de quatro pontos, sendo 0 (nenhuma vez) e 3 (quase todos os dias), cujo somatório da pontuação varia de 0 a 27 pontos. O escore total de 1-4 indica a depressão mínima, de 5-9, depressão suave, de 10-14, depressão moderada, de 15-19, depressão moderadamente grave e de 20-27, depressão grave. Além disso, este instrumento é utilizado para indicar depressão maior, quando a pontuação total fica igual ou superior a 10 pontos $^{(18-19,25)}$

A coleta de dados foi realizada no ambiente universitário, mediante a realização do convite aos estudantes para participação na pesquisa. Posteriormente, as pesquisadoras explicitaram sobre a pesquisa, leram o Termo de Consentimento Livre e Esclarecido (TCLE) e àqueles que aceitaram, foi solicitado a assinatura do TCLE e entregue os questionários.

\section{Análise dos dados}

Os dados coletados foram analisados através do software Statistical Packcage for Social Science (SPSS) na versão 21.0. As variáveis categóricas foram expressas como frequência absoluta e, as porcentagens e as variáveis contínuas foram analisadas com mediana e intervalo interquartil, a depender da natureza da variável. O teste do qui-quadrado foi utilizado para identificar a associação das variáveis sociodemográficas e acadêmicas com os níveis de severidade da depressão. 
Adotaram-se, como estatisticamente significativas, as associações com o $\mathrm{p}<0,05$.

\section{Aspectos éticos}

O estudo foi aprovado pelo Comitê de Ética Institucional sob o Parecer: 3.193.785, CAAE: 08598819.4.0000.5056. Todos os participantes leram e assinaram o TCLE.

\section{Resultados}

Participaram do presente estudo 521 estudantes universitários, com mediana de idade de 21 anos, variando de 19 a 23 anos. A maioria eram mulheres 347 (66,6\%), $259(49,7 \%)$ eram solteiros, $117(21,7 \%)$ exerciam alguma atividade laboral e 258 cursavam os semestres iniciais da graduação. No que diz respeito aos cursos, os participantes apresentaram maior frequência para o curso de Enfermagem 147 (26,7\%), seguido de Direito $110(20,0 \%)$ e de Odontologia $72(13,1 \%)$.

Com relação à distribuição da depressão entre os estudantes, 163 (31,3\%) foram classificados como indivíduos com depressão suave, 122 (23,4\%) com depressão mínima, $100(19,2 \%)$ com depressão moderada, $68(13,1 \%)$ com depressão moderadamente grave e $50(9,6 \%)$ com depressão grave (Figura 1 ).

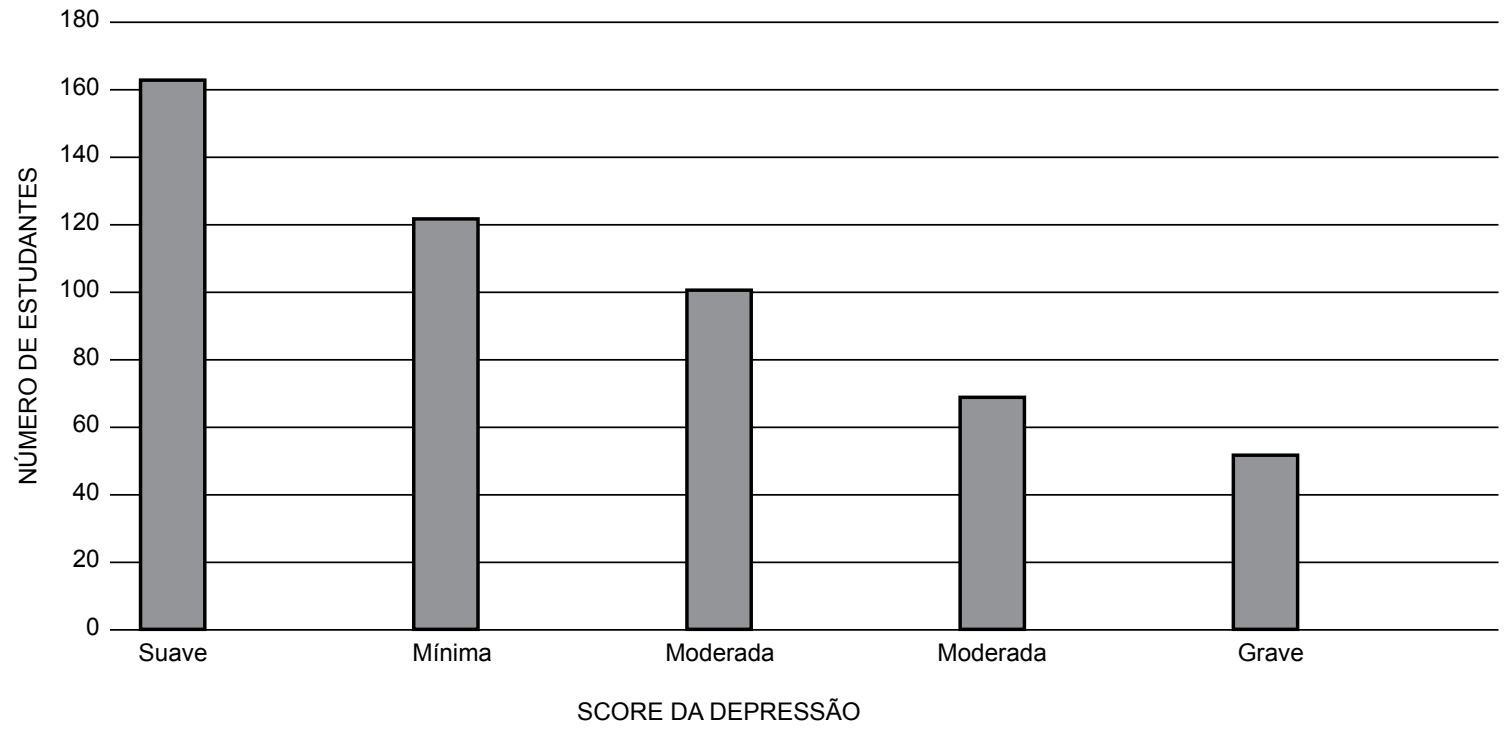

Figura 1 - Frequência de depressão em estudantes universitários de acordo com a classificação da escala PHQ-9, Brasília, DF, Brasil, 2019

No que diz respeito à associação dos fatores sociodemográficos e acadêmicos, com os níveis de severidade da depressão, constatou-se que, a renda familiar e o semestre apresentaram significância estatística (Tabela 1).

Tabela 1 - Associações das variáveis sociodemográficas e acadêmicas com o nível de severidade dos sintomas depressivos em estudantes universitários. Brasília, DF, Brasil, 2019

\begin{tabular}{lccc}
\hline \multicolumn{1}{c}{ Variáveis } & \multicolumn{2}{c}{ Depressão- $\mathbf{n}(\%)$} & \multirow{2}{*}{$\mathbf{p}^{*}$} \\
\cline { 1 - 2 } $\begin{array}{l}\text { Mínimo/Leve } \\
\text { Fexo }\end{array}$ & $\begin{array}{c}\text { Moderado/ } \\
\text { Grave }\end{array}$ & 0,137 \\
Masculino & $171(63,1)$ & $165(71,1)$ & \\
Relacionamento & $96(35,4)$ & $63(27,2)$ & \\
Solteiro & & & 0,310 \\
Namorando & $133(49,1)$ & $121(52,2)$ & \\
Casado & $80(29,5)$ & $70(30,2)$ & \\
Outros ${ }^{\dagger}$ & $21(7,7)$ & $13(5,6)$ & \\
\hline & $36(13,3)$ & $27(11,6)$ & (continua...)
\end{tabular}

\begin{tabular}{|c|c|c|c|}
\hline \multirow[t]{2}{*}{ Variáveis } & \multicolumn{2}{|c|}{ Depressão- n (\%) } & \multirow[b]{2}{*}{$\mathbf{p}^{*}$} \\
\hline & Mínimo/Leve & $\begin{array}{c}\text { Moderado/ } \\
\text { Grave }\end{array}$ & \\
\hline Profissão & & & 0,700 \\
\hline Estudante & $198(73,1)$ & $159(68,5)$ & \\
\hline $\begin{array}{l}\text { Estudante/ } \\
\text { Trabalhador }\end{array}$ & $51(18,8)$ & $56(24,1)$ & \\
\hline Outros $^{\ddagger}$ & $20(7,4)$ & $17(7,4)$ & \\
\hline $\begin{array}{l}\text { Renda } \\
\text { individual(salário } \\
\text { mínimo 2019- } \\
\mathrm{R} \$ 998,00)\end{array}$ & & & 0,663 \\
\hline $\begin{array}{l}\text { Até um salário } \\
\text { mínimo }\end{array}$ & $62(22,9)$ & $65(28,0)$ & \\
\hline $\begin{array}{l}\text { De } 01 \text { a } 03 \text { salários } \\
\text { mínimos }\end{array}$ & $55(20,3)$ & $51(22,0)$ & \\
\hline $\begin{array}{l}\text { De } 03 \text { a } 05 \text { salários } \\
\text { mínimos }\end{array}$ & $9(3,3)$ & $5(2,2)$ & \\
\hline $\begin{array}{l}\text { De } 05 \text { a } 15 \text { salários } \\
\text { mínimos }\end{array}$ & $3(1,1)$ & $1(0,4)$ & \\
\hline $\begin{array}{l}\text { Mais de } 15 \text { salários } \\
\text { mínimos }\end{array}$ & $2(0,7)$ & $0(0,0)$ & \\
\hline Sem renda familiar & $135(49,8)$ & $107(46,1)$ & \\
\hline
\end{tabular}


(Tabela 1 - continuação)

\begin{tabular}{|c|c|c|c|}
\hline \multirow[t]{2}{*}{ Variáveis } & \multicolumn{2}{|c|}{ Depressão- n (\%) } & \multirow[b]{2}{*}{$\mathbf{p}^{*}$} \\
\hline & Mínimo/Leve & $\begin{array}{c}\text { Moderadol } \\
\text { Grave }\end{array}$ & \\
\hline $\begin{array}{l}\text { Renda familiar(salário } \\
\text { mínimo 2019- } \\
\mathrm{R} \$ 998,00)\end{array}$ & & & 0,006 \\
\hline $\begin{array}{l}\text { Até um salário } \\
\text { mínimo }\end{array}$ & $19(7,0)$ & $25(10,8)$ & \\
\hline $\begin{array}{l}\text { De } 01 \text { a } 03 \text { salários } \\
\text { mínimos }\end{array}$ & $105(38,7)$ & $84(36,2)$ & \\
\hline $\begin{array}{l}\text { De } 03 \text { a } 05 \text { salários } \\
\text { mínimos }\end{array}$ & $70(25,8)$ & $68(29,3)$ & \\
\hline $\begin{array}{l}\text { De } 05 \text { a } 15 \text { salários } \\
\text { mínimos }\end{array}$ & $47(17,3)$ & $30(12,9)$ & \\
\hline $\begin{array}{l}\text { Mais de } 15 \text { salários } \\
\text { mínimos }\end{array}$ & $15(5,5)$ & $8(3,4)$ & \\
\hline Sem renda familiar & $8(3,0)$ & $12(5,2)$ & \\
\hline Curso & & & 0,418 \\
\hline Direito & $57(21,0)$ & $41(17,7)$ & \\
\hline Educação Física & $28(10,3)$ & $15(6,5)$ & \\
\hline Enfermagem & $61(22,5)$ & $71(30,6)$ & \\
\hline Farmácia & $15(5,5)$ & $12(5,2)$ & \\
\hline Fisioterapia & $24(8,9)$ & $23(9,9)$ & \\
\hline Odontologia & $37(13,7)$ & $29(12,5)$ & \\
\hline Outros(Crush)§ & $48(17,7)$ & $39(16,6)$ & \\
\hline Semestre & & & 0,004 \\
\hline $1^{a}$ & $66(24,4)$ & $37(15,9)$ & \\
\hline $2^{\circ}$ & $8(3,0)$ & $8(3,4)$ & \\
\hline $3^{\circ}$ & $60(22,1)$ & $52(22,4)$ & \\
\hline $4^{\circ}$ & $4(1,5)$ & $9(3,9)$ & \\
\hline $5^{\circ}$ & $25(9,2)$ & $18(7,8)$ & \\
\hline $6^{\circ}$ & $16(5,9)$ & $9(3,9)$ & \\
\hline $7^{\circ}$ & $42(15,5)$ & $28(12,1)$ & \\
\hline $8^{\circ}$ & $18(6,6)$ & $20(8,6)$ & \\
\hline $9^{\circ}$ & $21(7,7)$ & $36(15,5)$ & \\
\hline $10^{\circ}$ & $5(1,8)$ & $10(4,3)$ & \\
\hline
\end{tabular}

*Teste do qui-quadrado; ${ }^{+}$Outros = Administração, Arquitetura, Ciências Contábeis, Engenharia Civil, Gestão Ambiental, Medicina, Nutrição, Psicologia, Estudante/Estagiário, Estudante/Trabalhador/ Voluntário, Estudante/ Voluntário; §Outros (Crush) = União Estável

\section{Discussão}

Os resultados deste estudo causam uma preocupação, visto a alta frequência da depressão (96,6\%), nesta amostra, que variou quanto à severidade. Conforme apontado pela literatura, trata-se de um problema considerável, visto que, a depressão é uma doença incapacitante que, inclusive, pode levar ao suicídio(26). Embora nesta amostra, a maior frequência de classificação da depressão foi do nível suave, é imprescindível que a comunidade acadêmica manifeste a devida atenção a esta população, pelo risco de evolução deste nível para os mais graves da doença, conforme a classificação da escala PHQ-9(18).

Um estudo transversal, conduzido com 1245 estudantes, na Arábia Saudita mostrou que, 34\% dos participantes estavam com depressão suave, $26 \%$ com depressão mínima, 24,6\% com depressão moderada, $10,4 \%$ com depressão moderadamente grave e $5 \%$ com depressão grave ${ }^{(27)}$. Da mesma forma, o presente estudo, apresentou resultados semelhantes quanto ao percentual de classificação da depressão, sendo mais comuns na atual amostra, a depressão suave, que pode ser percebida por hábitos do cotidiano como polifagia e hiporexia, procrastinação, apatia, descuido com a higiene pessoal, vontade constante de dormir ou de ficar na cama e uso excessivo das redes sociais ${ }^{(14)}$.

Em contrapartida, o estudo transversal realizado por Andreas e Brunborg, com amostra de 1326 estudantes noruegueses constatou que, o escore de depressão mínima foi o mais frequente $(46,8 \%)$, sendo resultado distinto do observado nesta pesquisa(20). Vale destacar que a realidade destes países, nos múltiplos aspectos, difere da realidade brasileira, e mesmo assim, apresentam resultados semelhantes com o atual estudo.

No estudo transversal conduzido com 234 estudantes de medicina da Faculdade Pernambucana de Saúde, cujo objetivo foi determinar a prevalência de sintomas de depressão, verificou-se que 5,6\% dos participantes manifestaram sintomas sugestivos de depressão. Ademais, observou-se associação da prevalência de sintomas de depressão ao uso de drogas psicoativas e ilícitas, o que indica a necessidade de medidas de prevenção e diagnóstico precoce para a doença(21).

No Brasil, são poucas as ações de promoção/ prevenção da saúde mental executadas no ambiente universitário, entretanto, algumas IES têm levantado a discussão quanto aos assuntos relacionados à saúde mental e desenvolvido atividades de promoção/prevenção da saúde para este público alvo(28-29).

A exemplo, pode-se citar uma ação realizada pela Universidade do Estado do Rio Grande do Norte que, no ano de 2018, promoveu o projeto denominado Práticas Integrativas na Faculdade de Ciências da Saúde, cujo objetivo foi desenvolver atendimentos psicossociais semanais com psicopedagogas e psicólogas educacionais e clínicas ${ }^{(30)}$.

Outra experiência exitosa identificada na literatura foi a realizada pela Universidade Federal do Pernambuco, na qual utilizou-se da psicologia positiva, a fim de estimular os sentimentos do bem-estar, da felicidade, do prazer no público acadêmico(31). O objetivo principal da psicologia positiva é incentivar sentimentos positivos, ou seja, não tem por foco a minimização de sintomas psiquiátricos ou emoções negativas. Ocorre que, ao ter pensamentos positivos, o ser humano tende a diminuir os sentimentos negativos, como consequência(32).

Ao se pensar na saúde mental da população, profissionais da psicologia, do estado de Minas Gerais, criaram uma campanha denominada "Janeiro Branco", planejada e projetada para a promoção da saúde emocional dos cidadãos, buscando estratégias políticas, sociais e culturais para que o adoecimento emocional seja prevenido, conhecido e combatido por toda a população(33). 
A Universidade Federal do Rio Grande do Sul, também divulgou, por meio de estudos, exemplos de ações de promoção à saúde. A exemplo, a promoção de debates por meio de reuniões e rodas de conversa com o corpo discente e docente, sobre saúde mental na instituição, a fim de criar iniciativas para reduzir os processos patológicos mentais nesta população(30,34). Da mesma forma, nesta Instituição foi desenvolvido um material específico para o site institucional denominado "Saúde Mental", que reuniu várias instruções quanto ao reconhecimento de sinais/sintomas de sofrimento psíquico, além da indicação de locais para apoio psicológico. Outro projeto executado nesta organização, denominado "Pega Leve", desenvolvido pelo corpo discente, teve como ação o trabalho de estudantes que desempenharam o papel de "agentes de saúde mental", ao auxiliar os estudantes com problemas de ordem psíquica/emocional a encontrarem locais de apoio(30).

Estudos mostram que, estratégias de promoção de saúde, no âmbito acadêmico, influenciam positivamente para a melhora da saúde, reduzem comportamentos de risco e melhoram o ambiente físico e social dos estudantes. Ademais, são essenciais para que, estudantes percebam a importância de equilibrar suas atividades diárias com atividades de lazer, como técnicas de relaxamento diversificadas, interação com outros estudantes e dinâmicas lúdicas ${ }^{(22-24)}$.

Diante das experiências exitosas supracitadas, destaca-se a importância de ações direcionadas para promoção e prevenção da saúde. Conforme apontados nos resultados desse estudo, a renda familiar e o semestre são fatores que podem influenciar na depressão e na sua severidade. Compreende-se, portanto, que a identificação de tais fatores, é primordial para o embasamento das ações de prevenção e promoção da saúde mental, tendo em vista, que estes fatores são alguns dos nós críticos que levam à depressão.

Evidências mostram a associação dos fatores socioeconômicos com problemas de ordem mental, como a depressão. A exemplo, o estudo de Yohannes e colaboradores, cujo objetivo foi explorar o status socioeconômico de famílias que apresenta uma pessoa com transtorno mental grave (SMD; psicose ou transtorno bipolar) ou depressão em comparação com famílias sem uma pessoa afetada, os resultados apontaram que, as famílias que apresentavam algum membro com transtorno mental grave ou depressão, eram socioeconomicamente desfavorecidos, quando comparados à população geral. Do mesmo modo, um estudo realizado na Alemanha com 12484 participantes, com objetivo de analisar a associação transversal e longitudinal de três dimensões definidas do SES (escolaridade, posição ocupacional e renda líquida do domicílio) com a ocorrência de sintomas elevados de depressão em relação ao impacto de importantes covariáveis, observou que o status socioeconômico desempenha papel importante no desenvolvimento da severidade dos sintomas depressivos e que, quanto mais elevada a renda, menor o risco de desenvolver sintomas depressivos ${ }^{(35-36)}$.

Compreende-se que, a partir dos fatores identificados, mais estratégias de saúde, possam ser planejadas e executadas em diferentes comunidades e instituições de ensino e de saúde. Além disso, ressalta-se a importância desse levantamento, para a adequação de tais estratégias, de modo que, ações de saúde mental, sejam moldadas para a realidade de cada população e local.

Apesar de não ter sido um dos objetivos deste estudo, destaca-se que, a IES, onde foi realizada esta pesquisa, possui um núcleo de apoio ao discente, no que se refere ao acompanhamento psicossocial. Assim, acredita-se que os achados desse estudo, possam contribuir para as tomadas de decisões, referentes às ações planejadas para o perfil deste público universitário, com vistas a diminuir a sintomatologia depressiva e estimular a conscientização da comunidade acadêmica, sobre os fatores que levam à depressão.

Entretanto, vale destacar a necessidade de sensibilizar a população universitária, quanto à identificação dos sinais/sintomas depressivos, para a busca pelos serviços de apoio à saúde mental, pelos próprios estudantes e/ ou pelo encaminhamento dos mesmos por professores/ coordenadores dos cursos da IES.

Vale salientar que, o atual estudo, teve como limitação a perda de informações, visto que a coleta de dados foi realizada mediante a aplicação de questionário e, alguns estudantes deixaram algumas questões sem respostas.

\section{Conclusão}

Acredita-se que, a divulgação dos resultados desta pesquisa seja importante para o conhecimento, tanto da comunidade acadêmica, quanto da comunidade externa da IES, devido à alta frequência da depressão $(96,6 \%)$ e seus fatores associados, de modo a incentivar estas populações para discussões acerca da temática, bem como para o desenvolvimento/reflexão de ações de promoção e prevenção à saúde mental no ambiente universitário. Logo, é crucial, pensar conjuntamente maneiras eficazes de identificar indivíduos no contexto acadêmico, com problemas de ordem psíquica emocional e levantar as causas que levam a tais desordens, como a depressão, objeto do presente estudo.

Além disso, acredita-se ser necessário mais estudos sobre a frequência e os fatores associados à depressão em universitários, nas diferentes regiões do país, a fim de aumentar o embasamento teórico-cientifico a respeito da doença e assim, conscientizar a população sobre a 
importância de se falar no assunto, no intuito de prevenir complicações decorrentes da depressão, como o suicídio.

\section{Referências}

1. Organização Pan- Americana de Saúde/ Organização Mundial de Saúde. Com depressão no topo da lista de causas de problemas de saúde, OMS lança a campanha "Vamos conversar". [Internet]. 2017. [Acesso 30 out 2018]. Disponível em: <https://www.paho.org/bra/index. php?option $=$ com_content $\&$ view $=$ article\&id $=5385$ : comdepressao-no-topo-da-lista-de-causas-deproblemas-de-saude-oms-lanca-a-campanha-vamosconversar\&Itemid $=839>$.

2. Mao Y, Zhang N, Liu J, Zhu B, He R, Wang X. A systematic review of depression and anxiety in medical students in China. BMC Med Educ . 2019. Available from: <https://bmcmededuc.biomedcentral.com/ articles/10.1186/s12909-019-1744-2>. doi: https://doi. org/10.1186/s12909-019-1744-2

3. Muniz FWMG, Maurique LS, Toniazzo MP, Rösing CK, Casarin M.Female undergraduate dental students may present higher depressive symptoms: A systematic review. Oral Dis. 2019. Available from: < https://www. researchgate.net/publication/330094828_Female_ undergraduate_dental_students_may_present_higher_ depressive_symptoms_A_systematic_review $>$. doi: 10.1111 / odi.13029

4. Tam W, Lo K, Pacheco J. Prevalence of depressive symptoms among medical students: overview of systematic reviews. Med Educ. 2019. Available from: < https://pubmed.ncbi.nlm.nih.gov/30474128/>. doi: $10.1111 /$ medu. 13770

5. Tung YJ, Lo KKH, Ho RCM, Tam WSW. Prevalence of depression among nursing students: A systematic review and meta-analysis. Nurse Educ Today. 2018. Available from: <https://pubmed.ncbi.nlm.nih.gov/29432998/>. doi: 10.1016 / j.nedt.2018.01.009

6. Rotenstein LS, Ramos MA, Torre M, Segal JB, Peluso MJ, Guille C et al. Prevalence of Depression, Depressive Symptoms, and Suicidal Ideation Among Medical Students: A Systematic Review and Meta-Analysis. JAMA. 2016. Available from: <https://www.ncbi.nlm.nih.gov/ pmc/articles/PMC5613659/\# : :text=In\%20this\%20 meta\%2Danalysis\%2C\%20the,\%2C\%2015.7\%25\%20 sought\%20psychiatric\%20treatment.> doi: 10.1001/ jama.2016.17324

7. Lei XY, Xiao LM, Liu YN, Li YM. Prevalence of Depression among Chinese University Students: A MetaAnalysis. PLoS One. 2016. Available from: <https:// journals.plos.org/plosone/article?id=10.1371/journal. pone. 0153454 : : text $=$ The $\% 20$ results $\% 20$ of $\% 20$ this $\% 20$ meta, depression $\% 20$ in $\% 20$ university $\% 20$ students $\% 20$ worldwide $\% 2$ C $>$. doi: https://doi. org/10.1371/journal.pone.0153454
8. Samaranayake CB, Arroll B, Fernando AT.Sleep disorders, depression, anxiety and satisfaction with life among young adults: a survey of university students in Auckland, New Zealand. N Z Med J . [Internet]. 2014. [cited June 5 2020]. Available from: < https://pubmed. ncbi.nlm.nih.gov/25145302/>.

9. Shamsuddin K, Fadzil F, Ismail WSW, Shah AS, Omar K, Muhammad NA et al. Correlates of depression, anxiety and stress among Malaysian university students. Asian J Psychiatry. 2013. Available from:< https://www.sciencedirect.com/science/article/abs/pii/ S1876201813000592?via\%3Dihub>. doi: https://doi. org/10.1016/j.ajp.2013.01.014.

10. Fernandes MA, Vieira FE, Silva JS, Avelino FVSD, Santos JDM. Prevalence of anxious and depressive symptoms in university students from a public institution. Rev Bras Enferm. 2018. Available from: <http://www. scielo.br/scielo.php?script $=$ sci_arttext\&pid=S003471672018001102169\&lng=pt. >. doi: http://dx.doi. org/10.1590/0034-7167-2017-0752

11. Coryell W, Winokur G. Depressive disorders. Merck Manual - Diagnosis and treatment (MSD) - Version for healthcare professionals. [Internet]. 2016. [cited May 23 2019]. Available from: <https://www.msdmanuals. com/pt-br/profissional/transtornos-psiqui\%C3\%A1tricos/ transtornos-do-humor/transtornos-depressivos>.

12. Ebert DD, Buntrock C, Mortier P, Auerbach R, Weisel KK, Kessler RC et al. Prediction of major depressive disorder onset in college students. Depression and Anxiety. 2019. Available from: <https://onlinelibrary.wiley.com/ doi/epdf/10.1002/da.22867>. doi: 10.1002/da.22867

13. Liu CH, Stevens C, Wong SHM, Yasui M, Chen JA. The prevalence and predictors of mental health diagnoses and suicide among U.S. college students: Implications for addressing disparities in service use. Depression Anxiety. 2018. Available from: <https://onlinelibrary. wiley.com/doi/abs/10.1002/da.22830>. doi: https://doi. org/10.1002/da.22830

14. Leão AM, Gomes IP, Ferreira MJM, Cavalcanti LPG. Prevalence and Factors Associated with Depression and Anxiety among University Students in the Field of Health in a Large Urban Center in the Northeast of Brazil. Rev Bras Educ Med. 2018. Available from: <http://www.scielo.br/pdf/rbem/v42n4/19815271-rbem-42-4-0055.pdf>.doi: https://doi. org/10.1590/1981-52712015v42n4rb20180092

15. Flesch BD, Houvèssou GM, Munhoz TN, Fassa AG. Major depressive episode among university students in Southern Brazil. Rev Saúde Pública. 2020. Available from: < https://www.scielo.br/scielo.php?script=sci_artt ext\&pid=S0034-89102020000100210 > . doi: https://doi. org/10.11606/s1518-8787.2020054001540

16. Pasqualucci PL, Damaso LLM, Danila AH, Fatori D, Lotufo Neto F, Koch VHK. Prevalence and correlates of 
depression, anxiety, and stress in medical residents of a Brazilian academic health system. BCM Med. Educ. 2018. Available from: <https://bmcmededuc.biomedcentral. com/articles/10.1186/s12909-019-1621-z>. doi: https:// doi.org/10.1186/s12909-019-1621-z

17. Barbosa JG, Filho NA. Miranda IMO, Rezende LF, Tank $\mathrm{K}$, Zeuri E. Characterization of suicide idea in university students. Rev UNINCOR. Universidade Vale do Rio Verde. 2018. Available from:< http://periodicos.unincor.br/ index.php/revistaunincor/article/view/3731/pdf_788>. doi: http://dx.doi.org/10.5892/ruvrd.v16i1.3731.g3426 18. Kroenke K, Spitzer RL, Williams JBW. The PHQ9: validity of a brief depression severity measure. Patient Health Questionnaire (PHQ) Screeners. JGIM. J Gen Internal Med. 2001. Available from: <https://onlinelibrary.wiley.com/doi/full/10.104 6/j.1525-1497.2001.016009606.x>. doi: https://doi. org/10.1046/j.1525-1497.2001.016009606.x

19. Ang AL, Wahab S, Abd Rahman FN, Hazmi H, Md Yusoff R. Depressive symptoms in adolescents in Kuching, Malaysia: Prevalence and associated factors. Pediatr Int. 2019. Available from: <https://onlinelibrary.wiley. com/doi/abs/10.1111/ped.13778>. doi: https://doi. org/10.1111/ped.13778

20. Andreas JB, Brunborg GS. Depressive Symptomatology among Norwegian Adolescent Boys and Girls: The Patient Health Questionnaire-9 (PHQ-9) Psychometric Properties and Correlates. Front Psychol., Department of Substance Use, Norwegian Institute of Public Health. [Internet]. 2017. [cited May 5 2019]. Available from: <https://www. frontiersin.org/articles/10.3389/fpsyg.2017.00887/full>. doi: https://doi.org/10.3389/fpsyg.2017.00887

21. Andrade LR, Barbosa L, Dias BRT, Melo GF, Souza E, Vasconcelos TC. Prevalence of Anxiety and Depression Symptoms among Medicine Students. Rev Bras Educ Med. 2015;39(1):135-42. Available from: <http://www.scielo.br/scielo.php?script=sci_arttext\& pid=S0100-55022015000100135>. doi: https://doi. org/10.1590/1981-52712015v39n1e00042014

22. Ferreira FMPB, Brito IM, Santos MR. Health promotion programs in higher education: integrative literature review. Rev Bras Enferm. 2018. Avalaible from:< https://www.scielo.br/pdf/reben/v71s4/pt_00347167-reben-71-s4-1714.pdf>. doi: http://dx.doi. org/10.1590/0034-7167-2016-0693

23. Braga ALS, Cavalcanti TVC, Cortez EA, Dutra VFD, Marinho TG, Mattos MMGR, et al. Promotion of Mental Health for University Students. Rev Pró UniverSUS. [Internet]. 2017. [cited April 25 2020]. Avalaible from:< http://editora.universidadedevassouras.edu.br/index.php/ RPU/article/view/896<.

24. Sahão FT. University student mental health: behaviors that favor adaptation to teaching higher. Universdade Estadual de Londrina. [Internet]. 2019. [cited May
25 2020]. Avalaible from:< http://www.uel.br/pos/ pgac/wp-content/uploads/2019/08/Saude-mental-doestudante-universitario-comportamentos-que-favorecema-adaptacao-ao-ensino-superior.pdf>.

25. Wondimagegnehu A, Abebe W, Abraha W, Teferra S. Depression and social support among breast cancer patients in Addis Ababa, Ethiopia. BMC Cancer. 2019. Avalaible from: < https://bmccancer.biomedcentral.com/ articles/10.1186/s12885-019-6007-4>. doi: https://doi. org/10.1186/s12885-019-6007-4

26. Melo AK, Siebra AJ, Moreira V. Depression in Adolescents: Review of the Literature and the Place of Phenomenological Research. Psicol Cienc Prof. 2017. Available from:< http:// www.scielo.br/scielo.php?script $=$ sci_arttext\&pi d=S1414-98932017000100018>. doi: http://dx.doi. org/10.1590/1982-37030001712014

27. Alharbi R, Alsuhaibani K, Almarshad A, Alyahia A. Depression and anxiety among high school student at Qassim Region. J Family Med Prim Care. 2019. Available from: <https://www.ncbi.nlm.nih.gov/pmc/articles/ PMC6436297/>.doi: 10.4103 / jfmpc.jfmpc_383_18

28. Santos JC. Como enfrentar o adoecimento mental na universidade: conheça iniciativas de professores e alunos [Internet]. Rio Grande do Sul (RS): Universidade Federal do Rio Grande do Sul; 2019 [Acesso 8 nov 2019]. Disponível em: <https://www.ufrgs.br/humanista/2019/10/10/ como-enfrentar-o-adoecimento-mental-na-universidadeconheca-iniciativas-de-professores-e-alunos/>.

29. Robaert DW, Schonardie EF. Education and practices for the promotion of mental health in. RC\&E. 2017; 32(103):191-212. Available from: <http://dx.doi.org/10.21527/21791309.2017.103.191-212>. doi: https://doi. org/10.21527/2179-1309.2017.103.191-212

30. Morais A. PRAE desenvolve projetos voltados à saúde mental dos estudantes [Internet]. Rio Grande do Norte (RN): Universidade do Estado do Rio Grande do Norte; 2018 [Acesso 16 nov 2019]. Disponível em: < [http:// portal.uern.br/blog/prae-desenvolve-projetos-voltadosa-saude-mental-dos-estudantes/>.

31. Victor JF, Lopes MVO, Ximenes LB. Analysis of diagram the health promotion model of Nola J. Pender. Acta Paul Enferm. 2005; 18(3):235-40. Available: <http:// www.scielo.br/scielo.php?script=sci_arttext\&pid $=$ S010321002005000300002\&lng=en>. doi: https://doi. org/10.1590/S0103-21002005000300002

32. Tavares LM. Common mental disorders and subjective wellbeing in medical students: a preventive intervention based on positive psychology. [Internet]. Recife (PE): Universidade Federal de Pernambuco; 2017 [cited Nov 16 2019]. Available from: <https://repositorio.ufpe.br/ handle/123456789/26477>. 
33. Abrahão L, Ribeiro V. Campanha Janeiro Branco. Janeiro Branco: por uma cultura da Saúde Mental. [Internet]. Minas Gerais. 2020. [Acesso 25 jan 2020]. Disponível em: <http://janeirobranco.com.br/ projeto-janeiro-branco/>.

34. Pureza JR, Kuhn CHC, Castro EK, Lisboa CSM. Positive psychology in Brazil: a systematic review. Rev Bras Ter Cogn. [Internet]. 2012. [cited Jan 6 2020]. Available from: <http://pepsic.bvsalud.org/scielo.php?script=sci_ arttext\&pid=S1808-56872012000200006\&lng=pt\&nrm $=$ iso $>$.

35. Hailemichael Y, Hanlon C, Tirfessa K, Docrat S, Alem A, Medhin G et al. Mental health problems and socioeconomic disadvantage: a controlled household study in rural Ethiopia. Int J Equity Health. [Internet]. 2019. [cited June 1 2020]. Available from: <https://pesquisa. bvsalud.org/portal/resource/pt/mdl-31366362>.

36. Schlax J, Jünger $C$, Beutel ME, Münzel T, Pfeiffer $\mathrm{N}$, Wild $\mathrm{P}$, et al. Income and education predict elevated depressive symptoms in the general population: results from the Gutenberg health study. BMC Public Health. [Internet]. 2019. [cited June 1 2020]. Available from: <https://pesquisa.bvsalud.org/portal/resource/pt/ mdl-31014301>.

\section{Contribuição dos Autores}

Concepção e planejamento do estudo: Larissa Barreto dos Santos, Karina Gomes do Nascimento, Andréia Guedes Oliva Fernandes e Talita de Cássia Raminelli da Silva. Obtenção dos dados: Larissa Barreto dos Santos, Karina Gomes do Nascimento, Andréia Guedes Oliva Fernandes e Talita de Cássia Raminelli da Silva. Análise e interpretação dos dados: Larissa Barreto dos Santos, Karina Gomes do Nascimento, Andréia Guedes Oliva Fernandes e Talita de Cássia Raminelli da Silva. Análise estatística: Larissa Barreto dos Santos, Karina Gomes do Nascimento, Andréia Guedes Oliva Fernandes e Talita de Cássia Raminelli da Silva. Redação do manuscrito: Larissa Barreto dos Santos, Karina Gomes do Nascimento, Andréia Guedes Oliva Fernandes e Talita de Cássia Raminelli da Silva. Revisão crítica do manuscrito: Larissa Barreto dos Santos, Karina Gomes do Nascimento, Andréia Guedes Oliva Fernandes e Talita de Cássia Raminelli da Silva.

Todos os autores aprovaram a versão final do texto. Conflito de interesse: os autores declararam que não há conflito de interesse.
Recebido: 16.03 .2020

Aceito: 20.07 .2020 para fins comerciais, os usuários não têm de licenciar esses trabalhos derivados sob os mesmos termos. 